\title{
Las personas asintomáticas con adenomas tubulares pequeños no se beneficiarían con una colonoscopia
}

Is colonoscopy indicated for small adenomas found by screening flexible sigmoidoscopy? Wallace MB, Kemp JA, Trnka YM et al. Ann Intern Med. 1998;129:273-278.

\section{Objetivo}

Determinar la prevalencia de pólipos avanzados en el colon proximal en los pacientes que presentan adenomas pequeños en la sigmoidoscopia de rastreo.

\section{Diseño}

Estudio de cohorte prospectivo.

\section{Lugar}

Veterans Affairs Medical Center, Massachusetts, EE.UU.

\section{Pacientes}

Pacientes asintomáticos mayores de 50 años sin factores de riesgo para cáncer colorrectal.

\section{Descripción de los Tests}

Al momento de la sigmoidoscopia todos los pólipos hallados fueron biopsiados y a estos pacientes se les aconsejó una colonoscopia. Fueron considerados avanzados aquellos pólipos mayores de $10 \mathrm{~mm}$ o con componente tubulo-velloso, velloso o maligno. Se determinó la prevalencia y características de las lesiones proximales con la colonoscopía.

\section{Resultados Principales}

Entre los 4490 pacientes sometidos a sigmoidoscopia se detectaron $401(8.9 \%)$ con pólipos, de los cuales 301 (75\%) fueron estudiados posteriormente con una colonoscopia.

De los 90 pacientes con adenomas tubulares únicos de entre 1 a 5 $\mathrm{mm}$ en el colon distal, $0 \%$ (IC 95\% 0-4\%) presentaron pólipos avanzados en el colon proximal comparados con el $5.4 \%$ (IC $95 \%$ 2.4-10.4\%) hallado en los pacientes con pólipos proximales múltiples o de 6 a $10 \mathrm{~mm}$. y 7.9\% (IC95\% 2.6-17.6\%) con pólipos proximales avanzados. ( $p=0.013$ para la tendencia). Los pacientes de bajo riesgo (con pólipos únicos de 1 a $5 \mathrm{~mm}$ ) representaron el $44 \%$ del total de los casos que presentaron lesiones distales en la sigmoidoscopía de rastreo.

\section{Conclùsiones}

Entre los pacientes sometidos a una sigmoidoscopia de rastreo aquellos en los que se encuentran adenomas únicos menores a 5 $\mathrm{mm}$ tienen una baja prevalencia de pólipos proximales avanzadas y por lo tanto estos pacientes no se beneficiarían con una colonoscopia.

\section{COMENTARIO}

En los actuales algoritmos de rastreo la sigmoidoscopia sirve como "filtro" para indicar la colonoscopia. Esta estrategia se basa en la presunción de que las prevalencias de lesiones proximales y distales están asociadas. Sin embargo algunos autores informan igual incidencia de lesiones proximales en pacientes con y sin lesiones distales. En los pacientes con cánceres proximales la incidencia de lesiones distales es de sólo $17-35 \%$. Por lo tanto los adenomas rectosigmoideos no son marcadores sensibles para la mayoría de los cánceres proximales. Los más escépticos han comparado esta estrategia a la de rastrear el cáncer de mama efectuando la mamografía a una sola mama.

Varios estudios han encontrado que la prevalencia de lesiones proximales se incrementa con el aumento del tamaño, número y displasia de las lesiones distales ${ }^{1}$. En el presente estudio, la falta de un grupo control (estudio colonoscópico de pacientes con sigmoidoscopia nor$\mathrm{mal}$ ) impide comparar el riesgo de pacientes sin lesiones con aquellos con pólipos no avanzados y además establecer la sensibilidad de la sigmoidoscopia para detectar lesiones proximales.

Lógicamente, restringir la colonoscopia a los casos con pólipos distales avanzados aumenta el valor predictivo de la sigmoidoscopia, pero disminuye su sensibilidad, de por sí baja. Se estima que con esta estrategia sola se detectaría $10 \%$ de las neoplasias proximales.
Los autores concluyen que la prevalencia de lesiones proximales en pacientes con adenomas tubulares distales de hasta $5 \mathrm{~mm}$ es tan baja que no realizar una colonoscopia en este grupo reduciría sustancialmente los costos y no afectaría la efectividad del rastreo. Sin embargo esta estrategia implica una sigmoidoscopia flexible anual hasta $60 \mathrm{~cm}$ con resección y estudio anatomopatológico de todas las lesiones encontradas. Por otro lado, la realización de una colonoscopia total podría evitar tener que efectuar algún otro método de rastreo en los siguientes 10 años.

Es evidente que todavía se requiere una revisión en la forma de rastreo del cáncer colorrectal2 ${ }^{2}$. El desafío es desarrollar una estrategia que sea segura y efectiva para evaluar toda la extensión del colon y que además sea aceptable para los pacientes y los seguros médicos. Hasta el momento, la US Preventive Task Force ${ }^{3}$ recomienda (Recomendación tipo $\mathrm{B}^{*}$ ), realizar el rastreo a personas asintomáticas mayores de 50 años. Las dos estrategias sugeridas son la realización de sangre oculta en materia fecal en forma anual, y con el método de guayaco; 0 la realización periódica de una sigmoidoscopía flexible. Si entre el médico y el paciente eligen a la sigmoidoscopía como estrategia inicial, este estudio podría ayudar a limitar el estudio colonoscópico en paciente con pequeños adenomas distales.

*Ver Glosario

\section{Dr. Carlos A. Vaccaro}

Cirujano Especialista en Coloproctologia. Servicio de Cirugía General. Hospital Italiano de Buenos Aires.

\section{Referencias}

1. Schoen RE, Corle D, Cranson L et al. Is colonoscopy needed for nonadvanced adenoma found on sigmoidoscopy?. Gastroenterology 1998;115:533-541.

2. Winawer SJ, Fletcher RH, Miller L et al. Colorectal cancer screeening: clinical guidelines and rationale. Gastroenterology 1997;112:594-642.

3. Guide to Clinical Preventive Services. Second Edition. 1996 Williams \& Wilkins. Pp89-103. 